\title{
Relação entre o uso de metformina e a deficiência de vitamina B12 em pacientes com diabetes mellitus tipo 2
}

\author{
Relationship between metformin use and vitamin B12 deficiency in patients with type 2 \\ diabetes mellitus \\ Relación entre el uso de metformina y la deficiencia de vitamina B12 en pacientes con \\ diabetes mellitus tipo 2
}

Ana Cláudia Costa Pereira ${ }^{1 *}$, Marina Abreu Corradi Cruz¹, Camila Cardoso Barbosa ${ }^{2}$, Gabriela Tôrres Teixeira ${ }^{1}$, Gabriela Santos Perez ${ }^{1}$, Isabel Leste Machado ${ }^{1}$, Ivie Candida Freitas ${ }^{1}$, Julia Melo Coelho Lopes ${ }^{1}$, Luisa Argolo Assis ${ }^{1}$, Adriel Gustavo Lopes ${ }^{1}$.

\section{RESUMO}

Objetivo: Analisar a prevalência de pacientes com Diabetes Mellitus tipo 2 e hipovitaminose e compreender os impactos clínicos dessa correlação. Métodos: Foi acessado o DeCS para delimitar e selecionar os seguintes descritores: metformin, vitamin B12 e diabetes mellitus. Nas bases de dados PubMed, LILACS e SciELO, com auxílio de filtros de buscas, foram selecionados 20 artigos publicados nos últimos 10 anos. Resultados: O Diabetes Mellitus tipo 2 tem como primeira escolha no tratamento a metformina, que ajuda no controle glicêmico, auxilia na perda ponderal, reduz os riscos cardiovasculares e a mortalidade. Em contrapartida, existem evidências significativas da associação do uso de metformina à deficiência de vitamina B12. Esta hipovitaminose de cobalamina resulta da interferência na ação da membrana dependente de cálcio responsável pela absorção do fator intrínseco da vitamina B12 no íleo terminal, resultando em neuropatias e anemias megaloblásticas. Portanto, diante deste quadro, é importante realizar o controle vitamínico dos pacientes para a reposição assim que necessária. Considerações finais: Os estudos selecionados apresentaram uma alta prevalência de deficiência ou possível hipovitaminose em usuários de metformina. Por fim, é necessária a realização de novos estudos sobre os impactos clínicos dessa hipovitaminose para elucidar a necessidade da suplementação.

Palavras-chave: Diabetes mellitus, Metformina, Deficiência de vitamina B12.

\section{ABSTRACT}

Objective: To analyze the prevalence of patients with Type 2 Diabetes Mellitus and hypovitamin and understand the clinical impacts of this correlation. Methods: DeCS was accessed to define and select the following descriptors: metformin, vitamin B12 and diabetes mellitus. In the databases PubMed, LILACS and SciELO, with the aid of search filters, 20 articles published in last 10 years. Results: Type 2 Diabetes Mellitus feared as the first risk, not treatment with metformin, which does not control blood glucose, helps with weight loss, reduces cardiovascular risk and deaths. On the other hand, there is significant evidence of an association between the use of metformin and vitamin B12 deficiency. This cobalamin hypovitaminosis results from interference with a calcium-dependent membrane responsible for the absorption of intrinsic vitamin B12 from the non-terminal ileum, resulting in neuropathies and megaloblastic anemias. Therefore, for this reason, it is important to perform or monitor two patients for vitamin therapy as needed. Final considerations: The selected studies will have a high prevalence of deficiency or possible hypovitaminase in metformin users. Finally, further studies on the clinical impacts of hypovitamin are necessary to elucidate the need for supplementation.

Keywords: Diabetes mellitus, Metformin, Vitamin B12 deficiency.

\footnotetext{
${ }^{1}$ Pontifícia Universidade Católica de Minas Gerais (PUC-MG), Betim - MG.

*E-mail: anaclaudiacpereira@gmail.com

2 Faculdade UniAtenas, Sete Lagoas - MG.
} 


\section{RESUMEN}

Objetivo: Analizar la prevalencia de pacientes con diabetes mellitus tipo 2 e hipovitamina y comprender los impactos clínicos de esta correlación. Métodos: Se accedió a DeCS para definir y seleccionar los siguientes descriptores: metformina, vitamina B12 y diabetes mellitus. En las bases de datos PubMed, LILACS y SciELO, con la ayuda de filtros de búsqueda, se seleccionaron 20 artículos publicados en los últimos 10 años. Resultados: La diabetes mellitus tipo 2 temía como el primer riesgo, no el tratamiento con metformina, que no controla la glucosa en la sangre, ayuda a perder peso, reduce el riesgo cardiovascular y las muertes. Por otro lado, existe evidencia significativa de una asociación entre el uso de metformina y la deficiencia de vitamina B12. Esta hipovitaminosis de cobalamina resulta de la interferencia con una membrana dependiente de calcio responsable de la absorción de la vitamina B12 intrínseca del íleon no terminal, lo que resulta en neuropatías y anemias megaloblásticas. Por lo tanto, por esta razón, es importante realizar o controlar a dos pacientes para la terapia vitamínica según sea necesario. Consideraciones finales: Los estudios seleccionados tendrán una alta prevalencia de deficiencia o posible hipovitaminasa en usuarios de metformina. Finalmente, se necesitan más estudios sobre los impactos clínicos de la hipovitamina para dilucidar la necesidad de suplementación.

Palabras clave: Diabetes mellitus, Metformina, Deficiencia de vitamina B12.

\section{INTRODUÇÃO}

A diabetes mellitus (DM) é caracterizada por uma série de acometimentos sistêmicos que têm em comum o quadro hiperglicêmico (KUMAR V, et al., 2013). Esse quadro pode alterar a qualidade de vida do indivíduo, uma vez que se não for tratado adequadamente, pode gerar complicações macro e microvasculares, como cardiopatia isquêmica, acidente vascular cerebral, retinopatia e nefropatia. Além disso, apresenta alta prevalência, uma vez que 387 milhões de pessoas no mundo têm DM. Sendo assim, a prevenção e o tratamento precoce são de extrema importância para evitar complicações futuras e evitar prejuízos para o indivíduo, para a sociedade e para o sistema de saúde (MALTA DC, et al., 2019).

Os pacientes, geralmente assintomáticos, podem receber o diagnóstico da diabetes pela análise de alguns critérios, sendo eles: concentração aleatória de glicose de $200 \mathrm{mg} / \mathrm{dL}$ ou mais, com os sinais e sintomas clássicos; concentração de glicose de jejum de $126 \mathrm{mg} / \mathrm{dL}$ ou mais, em mais de uma ocasião; ou teste oral de tolerância à glicose (TOTG) anormal, no qual a concentração de glicose seja de $200 \mathrm{mg} / \mathrm{dL}$ ou mais, duas horas após uma a administração de $75 \mathrm{~g}$ de glicose. Cabe relembrar que os níveis séricos ideais variam de acordo com autores, mas considera-se euglicêmico, o indivíduo com valores entre 70-120 mg/dL (KUMAR V, et al., 2013).

Também é essencial analisar a importância do quadro hiperglicêmico pré-diabético prevalente, o qual se caracteriza por aumento dos níveis glicêmicos que não atingem os critérios absolutos para o diagnóstico de diabetes, mas representam elevado risco de desenvolvimento da patologia e devem ser acompanhados (KUMAR V, et al., 2013).

Sabe-se que a intervenção no estilo de vida do paciente é uma prática elegível neste caso, portanto recomenda-se que o paciente adote uma dieta hipocalórica para perda ponderal e pratique atividades físicas regularmente. Os resultados desta intervenção no estilo de vida, mesmo em pacientes de alto risco, são superiores ao uso de biguanidas como a metformina, por atrasar ou prevenir eficientemente o desenvolvimento da doença na população pré-diabética, promover qualidade de vida e postergar as complicações sistêmicas da diabetes sem oferecer riscos colaterais medicamentosos (DPPRG, 2015).

Os pacientes diabéticos, em sua maioria, se encaixam nas duas categorias mais prevalentes do diabetes mellitus: 0 diabetes tipo 1, que se caracteriza pela destruição das células beta pancreáticas e consequente deficiência da insulina ou o diabetes tipo 2 , definido pela resistência periférica à ação da insulina com a resposta compensatória inadequada das células beta pancreáticas, causada por influências genéticas e ambientais, como história familiar da doença, alimentação rica em carboidratos, sedentarismo, excesso de peso, hipertensão arterial, doença coronariana e uso de medicamentos hiperglicemiantes (TREVIZANI FA, et 
al., 2019). Esta última, segundo a Pesquisa Nacional de Saúde, realizada em 2013, apresenta-se no Brasil em $6,9 \%$ da população acima de 18 anos, sendo $7 \%$ mulheres e $5,4 \%$ homens, sendo, assim, o tipo mais prevalente de diabetes mellitus no país (NAZÁRIO A, et at., 2017).

Como tratamento dessa doença, além da mudança no estilo de vida, são usados os agentes hipoglicemiantes: metformina, sulfonilureias, inibidores da a-glucosidase e miméticos das incretinas de longa ação. Porém, a metformina é considerada a terapia de primeira escolha, devido a sua eficácia na diminuição da resistência à insulina e do risco cardiovascular e por ser bem tolerada, apesar dos efeitos colaterais transitórios como desconfortos gastrointestinais.

Este fármaco, classificado como biguanida, reduz a produção da glicose hepática - a gliconeogênese, que está aumentada de forma acentuada no diabetes tipo 2 -, aumenta a captação de glicose e utilização no músculo esquelético, reduz a absorção de carboidratos pelo intestino, aumenta a oxidação de ácidos graxos e reduz as lipoproteínas circulantes de baixa e alta densidades. Estes mecanismos garantem a diminuição dos níveis glicêmicos sem ocorrência de hipoglicemia e acidose láctica (RANG HP, et al., 2016). No entanto, apesar da eficácia conhecida e do perfil de segurança favorável, existem efeitos colaterais como a má absorção de vitamina B12 (DAMIÃO CP, et al., 2016).

Apesar de muita utilizada, a metformina traz consigo a dúvida se há maiores benefícios no uso precoce ou tardio do medicamento, bem como controversas opiniões sobre o uso na diabetes mellitus tipo 1. No diabetes tipo 1, a metformina é parcialmente usada para perda ponderal em pacientes com sobrepeso ou obesos a fim de evitar o desenvolvimento de resistência insulínica no organismo. Especificamente para a diabetes tipo 2, cerca de $80 \%$ dos pacientes diagnosticados prontamente recebem a prescrição de metformina e, em todo 0 mundo, cerca de 120 milhões de pessoas usam o fármaco (AMERICAN DIABETES ASSOCIATION, 2016 apud. MORANTES-CABALLERO JA, et. al., 2017).

De modo geral, sua ampla utilização na diabetes tipo 2 ocorre por, além do controle glicêmico, diminuir o apetite - auxilia no controle ponderal de pacientes obesos -, diminuir a circunferência abdominal e possibilitar a combinação com medicamentos das classes sulfonilureias, glitazonas e insulina. Os resultados ponderais podem ser mantidos por mais de uma década em pacientes analisados, se persistir o uso do medicamento (DPPRG, 2012). Outras síndromes que acompanham a resistência insulínica são tratadas com a metformina, como síndrome dos ovários policísticos, esteatose hepática não alcoólica, diabetes gestacional e puberdade prematura (RANG HP, et. al., 2016).

A vitamina B12 é hidrossolúvel e derivada de produtos de origem animal, como carne vermelha, laticínios e ovos. O fator intrínseco é uma glicoproteína produzida pelas células parietais no estômago e necessária para a absorção de B12 no íleo terminal (ANKAR A e KUMAR A, 2020). O termo vitamina B12 engloba todas as formas de cobalamina ativas em humanos, como cianocobalamina, hidroxocobalamina, metilcobalamina $e$ 5-desoxiadenosilcobalamina (adenosil-Cbl). A cobalamina possui cobalto hidrossolúvel em sua composição, servindo como cofator para enzimas metabólicas importantes. As funções mais importantes da B12 são atuar no metabolismo de ácido graxo, aminoácido e na síntese de DNA. Essas reações são imprescindíveis para o bom funcionamento da eritropoiese e do sistema nervoso central (AHMED MA, 2016).

As principais causas de deficiência de B12 são autoimunes, má absorção ou insuficiência alimentar. A hipovitaminose de cobalamina é de extrema importância devido às consequências graves que pode causar no organismo em longo prazo. Estas incluem danos neurológicos, englobando distúrbios cognitivos e psiquiátricos, instabilidade da marcha, disfunção autonômica e neuropatia, que pode ser equivocadamente confundida com a neuropatia periférica diabética e levar a um tratamento errôneo (LUTHRA N, et al., 2020).

A associação da metformina com a deficiência de vitamina B12 tem sido estudada há um tempo, mas o mecanismo que leva a essa deficiência ainda não está totalmente esclarecido. Recentemente, a deficiência de vitamina $\mathrm{B} 12$ tem sido associada à neuropatia em pacientes diabéticos tratados com metformina (LONGO $\mathrm{SL}$, et al., 2019). Nota-se que a deficiência de B12 causa consequências importantes no organismo, e observando a existência de grande número de pacientes portadores de DM2 em uso desse medicamento, faz-se importante analisar a prevalência de pacientes com diabetes mellitus tipo 2 e hipovitaminose de B12 e compreender os impactos clínicos dessa correlação. 
Devido à alta quantidade de portadores de Diabetes Mellitus tipo 2 na população e, consequentemente, o uso abundante da metformina, é de extrema importância ter o conhecimento da prevalência entre o uso da droga e a redução de vitamina B12 para que seja melhor compreendido os impactos clínicos dessa correlação, sendo esse o objetivo do estudo. Os artigos encontrados a partir da busca, explicitada na metodologia, trouxeram alguns estudos que foram realizados a fim de mostrar a relação entre 0 uso de metformina e a deficiência de vitamina B12.

\section{MÉTODOS}

A partir da escolha do tema, foi acessado o Descritores em Ciências da Saúde (DeCS) para delimitar os descritores. Com isso, os seguintes descritores foram escolhidos: metformin, vitamin B12 e diabetes mellitus e suas traduções em português. Após esta conduta, os descritores foram colocados em três bases de dados: Medical Literature and Retrivial System online (PubMed), Literatura Latino-Americana e do Caribe em Ciências da Saúde (LILACS) e Scientific Electronic Library Online (SciELO).

No PubMed, foram colocados os descritores com a variante de adição. Com isso, o número inicial de artigos foi 158, mas com a utilização do filtro de tempo, para selecionar artigos com menos de 10 anos, texto completo gratuito, ensaio clínico e revisão sistemática, o número de artigos reduziu para 6 . A segunda base de dados utilizada para pesquisa foi a SciELO; os descritores foram colocados na pesquisa avançada com a variante de adição, o que resultou em 5 artigos. Com o uso de filtro de busca com artigos publicados a partir de 2016, o número de artigos reduziu para 3.

A última base de dados utilizada para a pesquisa foi o LILACS, na qual foram usados os descritores metformin e vitamin B12 na pesquisa avançada, com a variante de adição, o que resultou em 2 artigos. Destes, somente 1 estava disponível para a leitura completa.

A partir dessa amostra, 10 artigos selecionados nas 3 bases de dados, os pesquisadores continuaram o processo de seleção por meio da leitura dos artigos. Dessa forma, foi utilizado como fator de inclusão: Artigos com metodologia bem delimitada e descrita. E como critério de exclusão: Fuga ao tema proposto. Com isso, foram selecionados 8 artigos.

A fim de complementar a introdução do artigo em relação a questões que abordam a fisiopatologia do Diabetes Mellitus e a deficiência da vitamina B12, foram realizadas três buscas adicionais na base de dados Pubmed e uma na base de dados SciELO. A primeira busca utilizou o descritor, previamente pesquisado no DeCS, vitamin B12 deficiency, o que resultou em 406 artigos, com a adição dos filtros free full text e 1 year, 0 número de artigos reduziu para 178. Destes foram selecionados 2 artigos que abordavam de forma clara e pertinente a fisiologia da cobalamina e as consequências da deficiência desta para o organismo, sem correlacionar com alguma morbidade.

A segunda busca utilizou o descritor diabetes mellitus, o que resultou em 136.293 artigos. Subsequentemente, refinou a busca com os filtros free full text, 1 year e portuguese, reduzindo o número de artigos para 72. Destes foram escolhidos 2 artigos que tratavam sobre a prevalência do diabetes mellitus no Brasil e a fisiologia deste distúrbio.

A terceira busca na base de dados Pubmed contou com a utilização de dois descritores, diabetes mellitus e metformin, o que mostrou um total de 11.929 artigos. Em sequência foram adicionados os filtros de revisão sistemática e clinical trial, free full text e artigos dos últimos 10 anos, e isso diminuiu o número de artigos para 672. A partir disso foram escolhidos 4 artigos que abordavam sobre o uso da metformina no diabetes mellitus.

Por fim, foi feita uma busca na base de dados SciELO com os descritores diabetes mellitus e metformin, o que resultou em 117. Consecutivamente foram adicionados os filtros de ciência da saúde, artigo revisado e artigos nos últimos 10 anos, com isso o número de artigos reduziu para 13. Destes foi elegido 1 artigo que contextualizava os dados de uso da metformina e aplicações desta no diabetes mellitus.

A partir destas pesquisas, foram selecionados 19 artigos, e juntamente a essa seleção foram utilizados os livros Patologia Básica de Kummar et al. (2013) e Farmacologia de Rang e Dale (2016), para complementar as informações acerca da fisiopatologia do diabetes mellitus e da importância da vitamina B12. 
Figura 1 - Critérios de inclusão e exclusão estabelecidos.
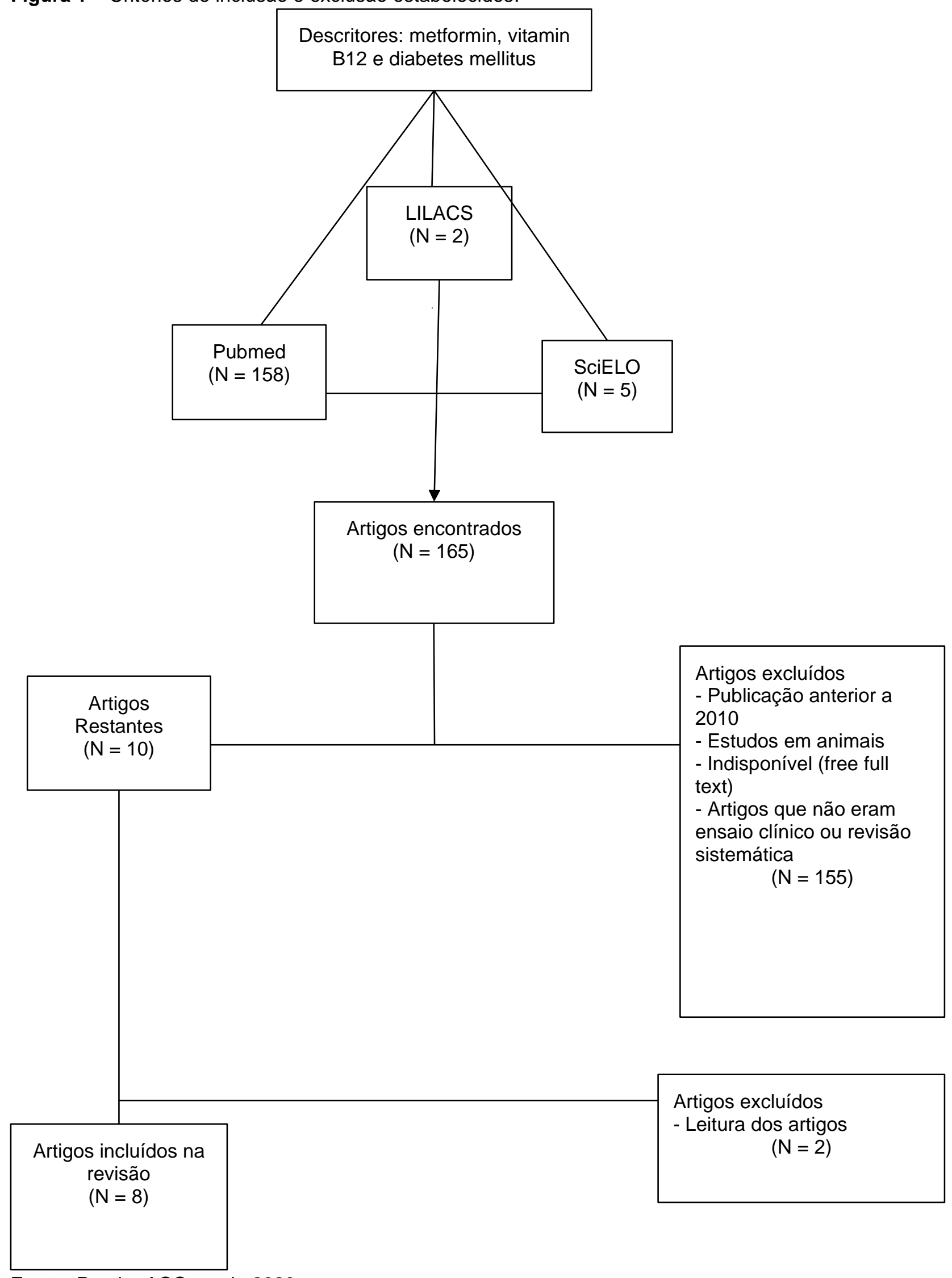

Fonte: Pereira ACC, et al., 2020. 


\section{Revista Eletrônica Acervo Saúde / Electronic Journal Collection Health | ISSN 2178-2091}

Quadro 1 - Artigos selecionados para o estudo.

\begin{tabular}{|c|c|c|}
\hline Autor/ano & Título & Principais informações \\
\hline 1- AHMED MA, et al. (2017) & $\begin{array}{c}\text { Perspectives on Peripheral Neuropathy as a } \\
\text { Consequence of Metformin-Induced Vitamin B12 } \\
\text { Deficiency in T2DM. }\end{array}$ & $\begin{array}{l}\text { Artigo de revisão que visa relacionar a neuropatia periférica como uma consequência da } \\
\text { deficiência de vitamina B12 induzida por metformina no DM2. A conclusão do artigo é que } \\
\text { a relação é conflitante, pois tanto a deficiência de vitamina B12 induzida por metformina e } \\
\text { a neuropatia periférica (NP) em pacientes com DM2, induzem neuropatias. Diferentes } \\
\text { perspectivas podem ser consideradas na interpretação e elaboração de estudos que } \\
\text { tentam explorar o triângulo da metformina, vitamina B12 e NP no DM2. }\end{array}$ \\
\hline 2- AHMED MA (2016) & $\begin{array}{c}\text { Metformin and Vitamin B12 Deficiency: where do we } \\
\text { stand? }\end{array}$ & $\begin{array}{l}\text { Artigo de revisão que visa estudar a correlação da hipovitaminose de B12 e o uso de } \\
\text { metformina. Há quase um consenso atual sobre o potencial da metformina para reduzir os } \\
\text { níveis de vitamina B12. Se o medicamento pode causar deficiência celular de vitamina B12 } \\
\text { permanece controverso. A prevalência relatada da deficiência apresentou grande variação } \\
\text { devido às diferenças nos biomarcadores diagnósticos utilizados, seus pontos de corte, bem } \\
\text { como fatores relacionados à amostra e ao estudo. Vários estudos investigaram } \\
\text { recentemente a capacidade da deficiência de vitamina B12 induzida por metformina em } \\
\text { causar ou piorar NP em pacientes com DM2, com resultados conflitantes. }\end{array}$ \\
\hline $\begin{array}{l}\text { 3- ANKAR A, KUMAR A. } \\
\text { (2019) }\end{array}$ & Vitamin B12 Deficiency (Cobalamin) & $\begin{array}{l}\text { Este artigo foca apenas na deficiência de vitamina B12, e seus aspectos gerais. A } \\
\text { hipovitaminose possui três etiologias primarias: autoimune, má absorção e insuficiência } \\
\text { alimentar. A epidemiologia da deficiência de vitamina B12 varia de acordo com a etiologia. } \\
\text { O artigo aborda também a fisiopatologia da doença, diagnósticos diferenciais, a avaliação } \\
\text { médica e possíveis complicações. }\end{array}$ \\
\hline 4- ARODA VR, et al. (2016) & $\begin{array}{l}\text { Long-term Metformin Use and Vitamin B12 Deficiency in } \\
\text { the Diabetes Prevention Program Outcomes Study }\end{array}$ & $\begin{array}{l}\text { O artigo tem como objetivo avaliar o risco de deficiência de B12 com o uso de metformina } \\
\text { na Prevenção do Diabetes Programa (DPP). } 3234 \text { participantes com tolerância à glicose } \\
\text { diminuída e jejum glicose no sangue de } 95 \text { a } 125 \mathrm{mg} \text { / dL, com pelo menos } 25 \text { anos de } \\
\text { idade e índice de massa corporal (IMC) de } 24 \mathrm{~kg} / \mathrm{m} 2 \text { ou superior. Os participantes foram } \\
\text { atribuídos aleatoriamente a um dos três tratamentos: placebo, um programa intensivo de } \\
\text { estilo de vida ou metformina } 850 \mathrm{mg} \text { duas vezes por dia. Todos os participantes assinaram } \\
\text { termo de consentimento. A análise dos níveis de vitamina B12 e homocisteína relatou um } \\
\text { risco aumentado de baixos níveis de vitamina B12 com tratamento a longo prazo com } \\
\text { metformina. Aos } 13 \text { anos desde a randomização e controle para idade, sexo, IMC basal, } \\
\text { supressão de ácido por prescrição terapia, diabetes e mudança de peso, houve um } 13 \% \\
\text { de aumento no risco de deficiência de vitamina B12 por ano de uso total de metformina. } \\
\text { Os níveis de homocisteína eram da mesma forma aumentou nos grupos com níveis de } \\
\text { vitamina B12 baixos ou limítrofes, apoiando a presença deficiência de vitamina B12 ao } \\
\text { nível do tecido. Além disso, naqueles tratados com metformina em comparação com } \\
\text { placebo, houve uma maior prevalência de anemia em média } 5 \text { anos após a randomização. }\end{array}$ \\
\hline
\end{tabular}

REAS/EJCH | Vol.12(10) | e4469 | DOI: https://doi.org/10.25248/reas.e4469.2020 Página 6 de 12 
Revista Eletrônica Acervo Saúde / Electronic Journal Collection Health | ISSN 2178-2091

\begin{tabular}{|c|c|c|}
\hline Autor/ano & Título & Principais informações \\
\hline 5- DAMIAO CP, et al. (2016) & $\begin{array}{l}\text { Prevalência de deficiência de vitamina B12 em pacientes } \\
\text { diabéticos do tipo } 2 \text { usando metformina: um estudo } \\
\text { transversal. }\end{array}$ & $\begin{array}{l}\text { O artigo tem como objetivo verificar a frequência de deficiência de vitamina B12 e seus } \\
\text { fatores relacionados em pacientes com diabetes mellitus tipo } 2 \text { em uso de metformina. Foi } \\
\text { observado que esses pacientes que usavam metformina com uso concomitante de } \\
\text { IBP/antagonistas-H2 tem maiores chances de desenvolver deficiência de vitamina B12 que } \\
\text { os não diabéticos. }\end{array}$ \\
\hline $\begin{array}{l}\text { 6- DPPRG - DIABETES } \\
\text { PREVENTION PROGRAM } \\
\text { RESEARCH GROUP (2015) }\end{array}$ & $\begin{array}{c}\text { Long-term effects of lifestyle intervention or metformin on } \\
\text { diabetes development and microvascular complications } \\
\text { over 15-year follow up: the Diabetes Prevention Program } \\
\text { Outcomes Study }\end{array}$ & $\begin{array}{l}\text { O artigo mostra a importância da prevenção eficaz do diabetes mellitus tipo } 2 \text { e os efeitos } \\
\text { benéficos que a mudança do estilo de vida dos pacientes ou do uso de metformina pode } \\
\text { acarretar. Foi feita essa avaliação durante o Programa de Prevenção do Diabetes (DPP) } \\
\text { que durou } 3 \text { anos e foi constatado no final que a intervenção no estilo de vida ou } \\
\text { metformina reduziu significativamente o desenvolvimento de diabetes ao longo de } 15 \text { anos. } \\
\text { Não houve diferenças no resultado microvascular entre os grupos de tratamento. No } \\
\text { entanto, aqueles que não desenvolveram diabetes tiveram uma prevalência menor de } \\
\text { complicações microvasculares do que aqueles que desenvolveram diabetes. Esse } \\
\text { resultado evidencia a importância da prevenção do diabetes. }\end{array}$ \\
\hline $\begin{array}{l}\text { 7- DPPRG - DIABETES } \\
\text { PREVENTION PROGRAM } \\
\text { RESEARCH GROUP (2012) }\end{array}$ & $\begin{array}{c}\text { Long-term safety, tolerability, and weight loss associated } \\
\text { with metformin in the Diabetes Prevention Program } \\
\text { Outcomes Study }\end{array}$ & $\begin{array}{l}\text { Esse artigo visa analisar se a metformina produziu perda de peso e retardou ou preveniu } \\
\text { o diabetes no Programa de Prevenção de Diabetes (DPP). O artigo compara um grupo } \\
\text { placebo com um grupo de pacientes em uso de metformina. O estudo mostrou uma maior } \\
\text { redução de peso e da circunferência de cintura nos pacientes em uso de metformina em } \\
\text { relação ao grupo placebo. No entanto, também foi observado mais sintomas } \\
\text { gastrointestinais nos pacientes em uso de metformina, mas esses sintomas foram } \\
\text { diminuindo com o tempo. }\end{array}$ \\
\hline 8- HASSAN N, et al. (2019) & $\begin{array}{c}\text { Association of Vitamin B12 Deficiency with Intake of Oral } \\
\text { Metformin in Diabetic Patients }\end{array}$ & $\begin{array}{l}\text { O artigo mostra um estudo transversal que tem como objetivo determinar o efeito do uso } \\
\text { de metformina no nível de vitamina B12. Foram incluídos no estudo } 72 \text { pacientes no total } \\
\text { e foram divididos em dois grupos: aqueles em uso de metformina por mais de dois anos } \\
\text { ( } 40 \text { pacientes) e os outros que não estavam usando metformina ( } 32 \text { pacientes). A } \\
\text { conclusão do artigo, após esse estudo foi de que o uso prolongado de metformina está } \\
\text { associado a deficiência de vitaminas B12. }\end{array}$ \\
\hline 9- JAGER J, et al. (2010) & $\begin{array}{l}\text { Long term treatment with metformin in type } 2 \text { diabetes } \\
\text { and risk of vitamin B-12 deficiency: randomised placebo- } \\
\text { controlled trial }\end{array}$ & $\begin{array}{l}\text { Este estudo foi parte de um estudo randomizado chamado Hyperinsulinemia:the Outcome } \\
\text { of your metabolic effects (HOME) que investigou os efeitos da metformina no metabolismo } \\
\text { e na doença microvascular e macrovascular. O ensaio inclui } 390 \text { pacientes com idade entre } \\
30-80 \text { anos com diabetes tipo } 2 \text { que estavam recebendo tratamento com insulina. Conclui } \\
\text { que pacientes com diabetes tipo } 2 \text { tratados com insulinas e aqueles tratados } \\
\text { adicionalmente com metformina tiveram um risco absoluto sete pontos percentuais maior } \\
\text { de deficiência de vitamina B12 do que aqueles tratados com placebo durante } 4,3 \text { anos de } \\
\text { acompanhamento. }\end{array}$ \\
\hline
\end{tabular}

REAS/EJCH | Vol.12(10) | e4469 | DOI: https://doi.org/10.25248/reas.e4469.2020 Página 7 de 12 


\section{Revista Eletrônica Acervo Saúde / Electronic Journal Collection Health | ISSN 2178-2091}

\begin{tabular}{|c|c|c|}
\hline Autor/ano & Título & Principais informações \\
\hline 10- LIU Q, et al. (2014) & $\begin{array}{c}\text { Vitamin B12 Status in metformin Treated Patients: } \\
\text { systematic review }\end{array}$ & $\begin{array}{l}\text { O artigo tem como objetivo mostrar que os ensaios clínicos randomizado e estudo } \\
\text { observacionais produziram resultados inconsistentes sobre os efeitos da metformina na } \\
\text { redução de vitamina B12. Portanto, realizou uma revisão sistemática para analisar os } \\
\text { efeitos da metformina na concentração de vitamina B12. }\end{array}$ \\
\hline 11- LONGO SL, et al (2019) & $\begin{array}{l}\text { Avaliação do monitoramento da vitamina B12 em } \\
\text { pacientes em uso de metformina em ambulatórios } \\
\text { urbanos. }\end{array}$ & $\begin{array}{l}\text { O artigo tem como objetivo avaliar a ocorrência de monitoramento dos níveis de vitamina } \\
\text { B12 em uma população diversificada. Conclui-se que maioria dos pacientes atendidos nos } \\
\text { locais de FQHC não tinha níveis de vitamina B12 monitorados, no entanto a maioria dos } \\
\text { pacientes monitorados tinha níveis normais de vitamina B12. Esse achado também pode } \\
\text { apoiar o monitoramento de pacientes com fatores de risco adicionais para deficiência de } \\
\text { vitamina B12, com uso simultâneo de medicamentos com outros agentes redutores de } \\
\text { vitamina B12 ou sintomas clínicos de deficiência, como neuropatia periférica. }\end{array}$ \\
\hline 12- LUTHRA N, et al. (2020) & $\begin{array}{l}\text { Vitamin B12 measurements across neurodegenerative } \\
\text { disorders. Journal of Clinical Movement Disorders }\end{array}$ & $\begin{array}{l}\text { Estudo transversal dos níveis da vitamina B12 no momento do diagnóstico de doença de } \\
\text { Parkinson e outros distúrbios neurodegenerativos. Pacientes com a doença de Parkinson } \\
\text { têm níveis de B12 mais baixos no momento do diagnóstico. Ainda são necessários mais } \\
\text { estudos para determinar se a deficiência dessa vitamina exerce influência nesses } \\
\text { distúrbios e em sua progressão. }\end{array}$ \\
\hline 13- MALTA DC, et al. (2019) & $\begin{array}{l}\text { Prevalência de diabetes mellitus determinada pela } \\
\text { hemoglobina glicada na população adulta brasileira, } \\
\text { Pesquisa Nacional de Saúde. Rev. bras. epidemiol. }\end{array}$ & $\begin{array}{l}\text { Calcularam-se as prevalências de diabetes em brasileiros por meio de análise dos dados } \\
\text { laboratoriais da Pesquisa Nacional de Saúde, coletados entre } 2014 \text { e } 2015 \text {. Sendo ela } \\
\text { maior no sexo feminino, acima de } 30 \text { anos, em população com baixa escolaridade, com } \\
\text { excesso de peso e obesidade. }\end{array}$ \\
\hline $\begin{array}{l}\text { 14- MORANTES- } \\
\text { CABALLERO JA, et al. } \\
\qquad(2017)\end{array}$ & $\begin{array}{c}\text { Metformina: más allá del control glucémico. Médicas UIS, } \\
\text { Bucaramanga }\end{array}$ & $\begin{array}{l}\text { Revisão bibliográfica que visa esclarecer e disseminar informações dos benefícios do uso } \\
\text { da metformina em pessoas com diabetes melittus além do controle glicêmico. Como, por } \\
\text { exemplo, reduzir a prevalência de insuficiência cardíaca, afetando a morbimortalidade e } \\
\text { como anticâncer. }\end{array}$ \\
\hline $\begin{array}{l}\text { 15- NAZÁRIO AR, et al. } \\
\text { (2017) }\end{array}$ & $\begin{array}{l}\text { Prevalência da deficiência de vitamina B12 em indivíduos } \\
\text { portadores de diabetes mellitus tipo } 2 \text { em uso de } \\
\text { metformina. Revista Sociedade Brasileira Clínica Médica }\end{array}$ & $\begin{array}{l}\text { Estudo transversal descritivo retrospectivo que, por meio de coleta de dados em } \\
\text { prontuários de pacientes atendidos no ambulatório de endocrinologia de um hospital em } \\
\text { Curitiba (PR), entre } 2014 \text { e } 2016 \text {, visa observar a prevalência de baixos níveis de vitamina } \\
\text { B12 em pacientes com diabetes mellitus tipo } 2 \text { em uso de metformina. A deficiência de } \\
\text { vitamina B12 foi prevalente nesses pacientes e devido às possíveis complicações, o } \\
\text { rastreio da deficiência e a suplementação vitamínica seria o ideal. }\end{array}$ \\
\hline
\end{tabular}

REAS/EJCH | Vol.12(10) | e4469 | DOI: https://doi.org/10.25248/reas.e4469.2020 Página 8 de 12 


\section{Revista Eletrônica Acervo Saúde / Electronic Journal Collection Health | ISSN 2178-2091}

\begin{tabular}{|c|c|c|}
\hline Autor/ano & Título & Principais informações \\
\hline 16- NERVO M, et al. (2011) & $\begin{array}{c}\text { Vitamina B12 em pacientes diabéticos tratados com } \\
\text { metformina: um estudo transversal no Brasil. Rev. Assoc. } \\
\text { Med. Bras. }\end{array}$ & $\begin{array}{l}\text { O objetivo desse estudo foi avaliar a prevalência de deficiência de vitamina B12 em } \\
\text { pacientes diabéticos tratados com metformina e os fatores associados aos níveis séricos } \\
\text { de vitamina B12.Nesse estudo foi usado método de corte transversal. Estes resultados } \\
\text { sugerem alta prevalência de deficiência de vitamina B12 em pacientes diabéticos tratados } \\
\text { com metformina. Pacientes mais velhos, em uso de metformina há muito tempo e com } \\
\text { ingestão baixa de vitamina B12 estão provavelmente mais predispostos a essa deficiência. }\end{array}$ \\
\hline $\begin{array}{l}\text { 17- SÁNCHEZ H, et al. } \\
\text { (2014) }\end{array}$ & $\begin{array}{l}\text { Vitamin B12 deficiency associated with high doses of } \\
\text { metformin in older people diabetic. Nutr. Hosp. }\end{array}$ & $\begin{array}{l}\text { Estudo caso-controle feito em pacientes diabéticos idosos com a finalidade de determinar } \\
\text { se o déficit de vitamina B12 associa-se com o uso de metformina. Os pacientes foram } \\
\text { divididos em dois grupos: Casos }(\mathrm{n}=137) \text { foram definidos como idosos com } \mathrm{B} 12<221 \\
\mathrm{pmol} / \mathrm{L} \text {; Controles ( } \mathrm{n}=279 \text { ) como idosos com } \mathrm{B} 12>221 \mathrm{pmol} / \mathrm{L} \text {. Quatro categorias de } \\
\text { uso de metformina foram definidas: não usuários, } \leq 850 \mathrm{mg} / \mathrm{dia} ;>850 \text { e }<2.550 \mathrm{mg} / \mathrm{dia} \text {; } \\
\geq 2.550 \mathrm{mg} / \text { dia. Doses elevadas de metformina }(\geq 2550 \mathrm{mg} / \mathrm{dia} \text { ) foram consideradas. } \\
\text { Observou-se que idosos que consumiram altas doses de metformina tiveram risco } 1,9 \\
\text { vezes maior de deficiência de B12, portanto estes resultados mostram uma forte } \\
\text { associação entre altas doses de metformina e baixos níveis de vitamina B12. }\end{array}$ \\
\hline $\begin{array}{l}\text { 18- TREVIZANI FA, et al. } \\
(2019)\end{array}$ & $\begin{array}{l}\text { Atividades de autocuidado, variáveis sociodemográficas, } \\
\text { tratamento e sintomas depressivos entre idosos com } \\
\text { Diabetes Mellitus. Revista Brasileira de Enfermagem. }\end{array}$ & $\begin{array}{l}\text { Estudo inferencial e transversal, com } 121 \text { idosos com Diabetes Mellitus Tipo } 2 \text { (DM) em } \\
\text { atendimento ambulatorial para analisar relação entre atividades de autocuidado de idosos } \\
\text { com DM tipo } 2 \text { e variáveis sociodemográficas, tipo de tratamento e sintomas depressivos. } \\
\text { Utilizaram-se Questionário para caracterização sociodemográfica e de saúde, } \\
\text { Questionário Atividades de autocuidado com o DM, Miniexame do Estado Mental e Escala } \\
\text { de Depressão Geriátrica. As maiores médias foram para as atividades "secar os espaços } \\
\text { entre os dedos dos pés, depois de lavá-los" e as menores para "prática de atividades } \\
\text { físicas". Apesar das médias elevadas para as atividades de autocuidado, observou-se a } \\
\text { necessidade de seu melhor desempenho e adesão. }\end{array}$ \\
\hline $\begin{array}{l}\text { 19- VALDES-RAMOS R, et } \\
\text { al. (2015) }\end{array}$ & $\begin{array}{c}\text { Vitamins and type } 2 \text { diabetes mellitus. Endocr Metab } \\
\text { Immune Disord Drug Targets. }\end{array}$ & $\begin{array}{l}\text { Revisão sistemática que avalia a relação entre diabetes mellitus tipo } 2 \text { e níveis de vitaminas } \\
\text { individuais ou combinadas. O estudo pontua que as vitaminas antioxidantes A, C e E são } \\
\text { encontradas naturalmente diminuídas em indivíduos diabéticos. A vitamina B12 foi vista } \\
\text { significativamente diminuída pelo uso prolongado de metformina. O estudo conclui que não } \\
\text { há nenhuma recomendação real sobre o uso de suplementos vitamínicos no diabetes } \\
\text { mellitus tipo } 2 \text { a ser emitida, no entanto, os pacientes que usam metformina durante } \\
\text { períodos prolongados podem precisar de ácido fólico e vitamina B12. }\end{array}$ \\
\hline
\end{tabular}

Fonte: Pereira ACC, et al., 2020.

REAS/EJCH | Vol.12(10) | e4469 | DOI: https://doi.org/10.25248/reas.e4469.2020 Página 9 de 12 


\section{RESULTADOS E DISCUSSÃO}

A correlação do uso da metformina no tratamento do Diabetes Mellitus e da deficiência de vitamina B12 é observada em 5 artigos que abordam estudos clínicos realizados. A partir disso, foi realizada uma análise individualmente desses artigos, abordando seus aspectos particulares, tais como, amostra, dose do medicamento, tempo do estudo e resultados finais.

Com o objetivo de observar a prevalência de baixas concentrações séricas de vitamina B12 em portadores de DM2 usuários de metformina, foi realizado um estudo transversal descritivo retrospectivo por meio de coletas de dados em prontuários de pacientes do ambulatório de endocrinologia em Curitiba (PR). Foram incluídos no estudo pacientes em uso de metformina por pelo menos 12 meses e que possuíam resultado de coleta sérica de B12. Além disso, foram excluídos pacientes que realizaram gastrectomia ou que fizeram suplementação de vitamina B12. Com isso, foram analisados 290 prontuários, sendo que 32,8\% apresentaram níveis séricos da vitamina $<200 \mathrm{pg} / \mathrm{ml}$ (significado: deficiência) e $36,9 \%$ entre 200 e $300 \mathrm{pg} / \mathrm{ml}$ (significado: possível deficiência).

Ademais, somente $30,3 \%$ foram diagnosticados com improvável déficit. A prevalência de deficiência conforme a dose do medicamento mostrou que dentre os pacientes que usaram metformina de 500 a $999 \mathrm{mg}$ diariamente, 5,1\% apresentaram deficiência de vitamina B12 e 10,3\% apresentaram possível deficiência, enquanto os pacientes que utilizaram doses mais elevadas, entre 1500 e $2550 \mathrm{mg}, 22,4 \%$ apresentaram deficiência e $22 \%$ possível deficiência. Ou seja, os achados indicaram alta prevalência de deficiência ou possível deficiência de vitamina B12 em diabéticos usuários de metformina (NAZÁRIO AR, et al., 2017).

Um outro estudo, com o objetivo de estabelecer a frequência de pacientes brasileiros portadores de DM2 em uso de metformina com deficiência de vitamina B12, foi realizado em um hospital universitário no sudeste do Brasil. O estudo contou com um total de 462 indivíduos, sendo um grupo controle (231 participantes) constituído por pessoas sem DM2 e o outro por pacientes portadores de DM2 em uso de metformina (231 pacientes). Os critérios de inclusão para o segundo grupo foram: possuir diagnóstico de DM2, ter pelo menos 18 anos e fazer uso de metformina por pelo menos 3 anos.

Já os critérios de exclusão, aplicados em ambos os grupos, foram: possuir história de gastrectomia, doenças mal-absorvidas, suplementação de B12 nos últimos 3 meses, anemia perniciosa, vegetariano e gestante. Todos os participantes foram submetidos a anamnese e a coleta de sangue. A deficiência de B12 (considerada $<170 \mathrm{pg} / \mathrm{ml}$ neste estudo) foi de $22,5 \%$ no grupo usuário de metformina, enquanto no grupo controle foi de $7,4 \%$. Dessa forma, concluiu-se que o tratamento prolongado de DM2 com metformina está associado a uma maior chance de desenvolver deficiência de B12 (DAMIÃO CP, et al., 2016).

Um terceiro estudo transversal foi realizado no ambulatório de Medicina Interna do Hospital de Clínicas de Porto Alegre com o objetivo de observar a prevalência de baixas concentrações séricas de vitamina B12 em portadores de DM2 usuários de metformina. Além disso, outros fatores também foram analisados como idade, sexo, peso, dosagem de metformina, duração do uso de metformina, ingestão alimentar de vitamina B12, volume corpuscular médio de eritrócitos (MCV), hemoglobina glicosilada e uso de outros medicamentos/suplementos.

Foram excluídos pacientes com histórico de gastrectomia, doença de Crohn, distúrbios do íleo terminal, suplementação de vitamina B12, uso irregular da metformina e aqueles que não realizaram a coleta de sangue. A análise foi feita com 144 pacientes diabéticos em uso da droga, em que 6,9\% da amostra apresentaram níveis séricos de B12 $<125 \mathrm{pg} / \mathrm{ml}$ (caracterizados como baixos) e $36,8 \%$ entre 125 e $250 \mathrm{pg} / \mathrm{ml}$ (caracterizados como possivelmente baixos). Com isso, os achados sugerem alta prevalência de redução de vitamina B12 em pacientes tratados com metformina (NERVO M, et al., 2010).

Outro ensaio clínico, realizado em três hospitais não acadêmicos na Holanda, foi feito com o objetivo de analisar os efeitos da metformina na incidência de deficiência da vitamina B12, considerada quando $<150$ $\mathrm{pg} / \mathrm{ml}$, ou da baixa concentração de vitamina B12, quando resultado entre 150 e 220pg/ml. Foi selecionada uma amostra de 390 pacientes diabéticos tipo 2 em tratamento com insulina, entre 30 e 80 anos de idade. Eles foram divididos aleatoriamente em 2 grupos, um para uso de metformina ( $850 \mathrm{mg}, 3$ vezes ao dia), outro para uso de placebo (3 vezes ao dia). 
Os medicamentos foram distribuídos em embalagens idênticas e o tratamento durou 4,3 anos. Foram coletadas amostras de sangue no início do estudo e após 4, 17, 30, 43 e 52 meses. Comparado ao placebo, o grupo que utilizou a metformina teve uma diminuição, em média, de 19\% da vitamina B12. O estudo, dessa forma, conclui que o tratamento prolongado com metformina aumenta o risco de deficiência de vitamina B12. Portanto, há indicação de medição regular do nível sérico de B12 (JAGER J, et al., 2010).

Mais um ensaio clínico, também com o objetivo de mostrar a deficiência da vitamina B12 quando utilizada a metformina para o tratamento e prevenção de DM2, principalmente a longo prazo, foi realizado em vinte e sete centros de estudos nos Estados Unidos. Os pacientes selecionados foram alocados de maneira randomizada em 3 grupos: tratamento com placebo (1082 pacientes), medidas de mudanças no estilo de vida e utilização da metformina $850 \mathrm{mg}$ duas vezes ao dia (1073 pacientes). Os níveis de B12 foram avaliados em 5 anos e em 13 anos. O resultado foi um aumento do risco de $13 \%$ de se desenvolver deficiência de vitamina B12 por ano do uso total de metformina. Ou seja, chegaram à mesma conclusão, que o uso prolongado por anos de metformina foi associado ao aumento do risco de deficiência de B12 (ARODA VR, et al., 2016).

\section{Impactos clínicos}

A metformina possui uma meia-vida de aproximadamente três horas e sua eliminação é de forma inalterada por filtração glomerular. O mecanismo de ação das biguanidas ainda não é muito claro, visto que ainda não se sabe quais são os alvos moleculares deste fármaco. No entanto, suas ações no organismo já são bem estabelecidas: reduz a produção de glicose hepática (gliconeogênese), a resistência insulínica, a absorção de carboidratos pelo intestino e a hiperlipidemia. Além disso, aumenta a captação de glicose, sua utilização no músculo esquelético e a oxidação de ácidos graxos (NAZÁRIO AR, et al., 2017).

Dessa forma, esse medicamento, além de aumentar a sensibilidade à insulina, reduz os riscos cardiovasculares e a mortalidade (RANG HP, et. al., 2016). Ademais, colabora na perda de peso, prevenindo o diabetes mellitus tipo 2 (NAZÁRIO AR, et al., 2017).

Outro benefício da metformina é que seus efeitos adversos são poucos e não incluem a hipoglicemia quando utilizada isoladamente. Porém, incluem distúrbios gastrointestinais dose dependentes, risco de acidose lática em pacientes com doença renal ou hepática, doença pulmonar hipóxica ou choque, que apesar de raros, são potencialmente fatais e deficiência de vitamina B12, interferindo em sua absorção (RANG HP, et. al., 2016).

A metformina é amplamente recomendada como agente de primeira linha para o tratamento da diabetes mellitus tipo 2, e tem efeitos benéficos no metabolismo de carboidratos, perda de peso e também na proteção vascular. Porém, como toda droga, possui efeitos colaterais importantes, e um dos mais observados é a correlação entre o uso de metformina e a queda da vitamina B12 no sangue (DAMIÃO CP, et al., 2016).

A explicação mais aceita atualmente para a hipovitaminose de cobalamina associada à metformina é que o medicamento interfere na ação da membrana dependente de cálcio responsável pela absorção do fator intrínseco da vitamina B12 no íleo terminal (ARODA VR, et al., 2016). Essa deficiência, em longo prazo, pode acarretar principalmente neuropatias e anemias megaloblásticas (NAZÁRIO AR, et al., 2017).

A neuropatia periférica é uma complicação primária do DM2, que se mostrou mais evidente em pacientes diabéticos com deficiência de vitamina B12, devido ao uso da metformina. No entanto, esse impacto não é facilmente percebido clinicamente em curtos períodos de uso do medicamento, uma vez que a hipovitaminose e suas consequências são de surgimento insidioso e prolongado (AHMED MA, 2016).

Já a anemia megaloblástica é uma condição causada pela redução no número de glóbulos vermelhos normais, que se tornam grandes, imaturos e disfuncionais na medula óssea. A principal causa da anemia megaloblástica é a redução da síntese de DNA na produção dos glóbulos vermelhos, que é uma das repercussões mais importantes da deficiência de vitamina B12. Alguns sintomas que podem estar associados são glossite, pancitopenia e disfunção gastrointestinal (AHMED MA, 2016).

É inquestionável que a hipovitaminose de cobalamina traz prejuízos à saúde, mesmo que só perceptíveis após longa exposição de metformina. Porém, como o medicamento é em sua maioria benéfico, é importante 
não a sua substituição, mas sim o controle vitamínico dos pacientes para a reposição assim que necessária. Porém, o diagnóstico da deficiência da vitamina B12 ainda é controverso, e não existe um teste padrão ouro, sendo difícil precisar a especificidade e sensibilidade dos exames existentes (AHMED MA, 2016).

Ainda que controverso sobre qual o melhor método, a dosagem é de suma importância, pois além de ser um tratamento simples, barato, benéfico, eficaz e seguro, quando detectado a tempo, pode evitar danos irreversíveis à saúde (NAZÁRIO AR, et al., 2017). Ademais, a monitoração do nível de B12 evita a suplementação desnecessária, uma vez que mesmo não sendo prejudicial e sendo barata, é um custo adicional financeiro para a saúde pública.

\section{CONSIDERAÇÕES FINAIS}

Feita uma revisão, chega-se à conclusão de que o uso de metformina para o tratamento de pacientes portadores de diabetes mellitus tipo 2 está diretamente relacionado à redução do nível sérico de vitamina B12. A maioria dos estudos apresentaram uma alta prevalência de deficiência ou possível deficiência de B12 em usuários dessa droga. Sendo assim, é necessário a realização de novos estudos analisando melhor os possíveis impactos clínicos dessa hipovitaminose na vida dos pacientes e a necessidade de um rastreamento sistemático dos níveis de vitamina B12 durante o uso da metformina, para que, assim, seja esclarecido se a suplementação deve ser prescrita ou não.

\section{REFERÊNCIAS}

1. AHMED MA, et al. Perspectives on Peripheral Neuropathy as a Consequence of Metformin-Induced Vitamin B12 Deficiency in T2DM. International Journal Of Endocrinology, 2017; 1-6.

2. AHMED MA. Metformin and Vitamin B12 Deficiency: where do we stand?. Journal Of Pharmacy \& Pharmaceutical Sciences, 2016; 19(3): 382-398.

3. ANKAR A, KUMAR A. Vitamin B12 Deficiency (Cobalamin). StartPearls Publishing, 2020.

4. ARODA VR, et al. Long-term Metformin Use and Vitamin B12 Deficiency in the Diabetes Prevention Program Outcomes Study. The Journal Of Clinical Endocrinology \& Metabolism, 2016; 101 (4): 1754-1761.

5. DAMIAO CP, et al. Prevalência de deficiência de vitamina B12 em pacientes diabéticos tipo 2 em uso de metformina: um estudo transversal. São Paulo Med. J, 2016; 134(6): 473-479.

6. DPPRG - Diabetes Prevention Program Research Group. Long-term effects of lifestyle intervention or metformin on diabetes development and microvascular complications over 15-year follow-up: the Diabetes Prevention Program Outcomes Study. Lancet Diabetes Endocrinol. 2015; 3(11): 866-875.

7. DPPRG - Diabetes Prevention Program Research Group. Long-term safety, tolerability, and weight loss associated with metformin in the Diabetes Prevention Program Outcomes Study. Diabetes Care, 2012; 35(4): 731-737.

8. HASSAN N, et al. Association of vitamin B12 Deficiency With Intake of oral Metformin In Diabetic Patients. J Ayub Med Cool Abbottabad, 2019.

9. JAGER J, et al. Long term treatment with metformin in patients with type 2 diabetes and risk of vitamin B-12 deficiency: randomised placebo controlled trial. Bmj, 2010; 340(194): 1-7.

10. KUMAR V, et al. Robbins patologia básica. 9. ed. Rio de Janeiro: Elsevier, 2013; 739p.

11. LIU Q, et al. Vitamin B12 Status in Metformin Treated Patients: systematic review. Plos One, 2014; 9(6): 1-6.

12. LONGO SL, et al. Avaliação do monitoramento da vitamina $B 12$ em pacientes em uso de metformina em ambulatórios urbanos. Pharmacy Pract (Granada), 2019; 17(3).

13. LUTHRA N, et al. Vitamin B12 measurements across neurodegenerative disorders. Journal of Clinical Movement Disorders, 2020.

14. MALTA DC, et al. Prevalência de diabetes mellitus determinada pela hemoglobina glicada na população adulta brasileira, Pesquisa Nacional de Saúde. Rev. bras. epidemiol., 2019.

15. MORANTES-CABALLERO JA, et al. Metformina: más allá del control glucémico. Medicas UIS, Bucaramanga, 2017; 30(1): $57-71$.

16. NAZÁRIO AR, et al. Prevalência da deficiência de vitamina B12 em indivíduos portadores de diabetes mellitus tipo 2 em uso de metformina. Revista Sociedade Brasileira Clínica Médica, 2017; 2(16): 99-103.

17. NERVO M, et al. Vitamina B12 em pacientes diabéticos tratados com metformina: um estudo transversal no Brasil. Rev. Assoc. Med. Bras., 2011; 57(1): 46-49.

18. RANG HP, et al. Rang \& Dale Farmacologia. 8. ed. Rio de Janeiro: Elsevier, 2016; 914

19. SANCHEZ H, et al. Vitamin B12 Deficiency Associated With Doses of Metformin in Older People Diabetic. Nutr Hosp, 2014; 29(6): 1394-1400.

20. TREVIZANI FA, et al. Atividades de autocuidado, variáveis sociodemográficas, tratamento e sintomas depressivos entre idosos com Diabetes Mellitus. Rev. Bras. Enferm., 2019; 72(2): 22-29.

21. VALDES-RAMOS R, et al. Vitamins and Type 2 Diabetes Mellitus. Endocrine, Metabolic \& Immune Disorders-drug Targets, 2015; 15(1): 54-63.

REAS/EJCH | Vol.12(10) | e4469 | DOI: https://doi.org/10.25248/reas.e4469.2020 Página 12 de 12 